Meta

Journal des tradlucteurs

Translators' Journal

\title{
Du caractère concret ou abstrait de textes néerlandais et français
}

\section{Marie-Jeanne de Vriendt-de Man}

Volume 15, numéro 4, décembre 1970

URI : https://id.erudit.org/iderudit/004581ar

DOI : https://doi.org/10.7202/004581ar

Aller au sommaire du numéro

Éditeur(s)

Les Presses de l'Université de Montréal

ISSN

0026-0452 (imprimé)

1492-1421 (numérique)

Découvrir la revue

Citer cet article

de Vriendt-de Man, M.-J. (1970). Du caractère concret ou abstrait de textes néerlandais et français. Meta, 15(4), 203-211. https://doi.org/10.7202/004581ar d'utilisation que vous pouvez consulter en ligne.

https://apropos.erudit.org/fr/usagers/politique-dutilisation/ 


\section{de textes \\ $\mathrm{Du}$ caractère concret ou abstrait $\bigcirc$ néerlandais et français}

Il existe dans le domaine de la stylistique comparée du français et d'une langue germanique deux ouvrages qui font autorité : celui de J.-P. Vinay et J. Darbelnet, Stylistique comparée du français et de l'anglais (Paris, éd. corrigée, Didier, 1964) et celui de A. Malblanc, Stylistique comparée du français et de l'allemand (Paris, Didier, 1963).

Jusqu'à présent l'analyse stylistique contrastive français/néerlandais n'a fait l'objet d'aucune étude approfondie. Cependant, celui qui est familiarisé avec la traduction du néerlandais au français, et vice versa, remarque que certaines différences, caractéristiques des couples français/anglais ou français/allemand, pourraient être observées dans la comparaison du français et du néerlandais.

Le néerlandais occupe une position intermédiaire entre l'anglais et l'allemand : il présente en effet des similitudes tantôt avec l'une de ces deux langues, tantôt avec l'autre. On pourrait dès lors chercher à déterminer dans quelle mesure les conclusions valables pour l'anglais ou l'allemand sont applicables au néerlandais en se laissant guider uniquement par sa connaissance et son intuition de la langue. Il me semble toutefois que cette manière de procéder n'est sûre que si elle est complétée par une observation objective de faits de langue dans un corpus même limité. L'intuition joue alors un rôle moins important et des similitudes qui ne sont qu'apparentes peuvent être décelées et écartées.

Le but de la présente étude est de déterminer si, comme l'allemand et l'anglais, le néerlandais s'exprime de façon plus concrète que le français. Un texte néerlandais, lorsqu'il est traduit en français, devient-il plus «abstrait »? Un texte français devient-il plus «concret» lorsqu'il est traduit en néerlandais?

Afin de travailler sur un ensemble homogène, $j$ 'ai analysé uniquement des extraits de textes littéraires; j'ai donc provisoirement écarté des textes scientifiques et techniques. Celui qui désire comparer des textes littéraires avec leurs traductions choisit ses extraits en tenant compte non seulement du genre de l'œuvre, mais aussi de la personnalité de l'auteur et même de celle du traducteur. 
C. Buysse, E. Claes, F. de Pillecijn et J. Vandeloo jouissent, pour des raisons diverses, de la faveur des lecteurs néerlandophones. Que de surcroît des traducteurs de talent tels que P. Brachin, M. Buysse, L. Fessard et H. Plard aient traduit en tout ou en partie l'œuvre de ces auteurs, m'a paru être un motif suffisant pour en étudier des extraits ${ }^{1}$.

Comparant les deux modes de représentation propres au français et à l'allemand, A. Malblanc écrit : «Nous avons tout d'abord reconnu deux grands plans sur lesquels se meuvent de préférence les deux représentations, l'allemand sur le plan du réel, le français sur le plan de l'entendement, proximité du concret pour l'un, tendance à la généralisation pour l'autre... ${ }^{2} \gg$. Dire que l'expression allemande est plus concrète que l'expression française équivalente est vague. A. Malblanc montre que l'expression concrète est liée à la réalité, à une forme, à un objet matériel qui peut être appréhendé par les sens. Contrairement à l'expression française qui relève de l'entendement, du monde des idées et qui est générale, l'expression allemande est plus précise parce qu'elle s'attache au détail.

Est-ce que ces constatations valent aussi pour l'expression néerlandaise? Quelles sont les caractéristiques d'un syntagme néerlandais « concret»?

a) Le verbe néerlandais est plus imagé et plus spécifique que son équivalent français :

Hij kon hem niet thuisbrengen... (Vandeloo, p. 198)

Impossible de le classer... (Buysse)

En na de derde dag kwam het nieuws tot hen door... (Pillecijn, p. 76)

Et au bout de trois jours la nouvelle leur arriva... (Brachin)

Il est plus suggestif; il est aussi chargé de nuances affectives que le verbe français n'a pas à cause de sa généralité.

De trein kwam nu langzaam op gang en liet het perron los. (Vandeloo, p. 200)

Lentement, le train s'ébranle et quitte le quai. (Buysse)

Onmiddellijk flapte zij haar Vlaams patois er weer tussendoor... (Buysse)

Mais [elle] y entremêlait aussitôt son patois flamand... (Fessard)

Waar steekt hij zijn snuit in? (Vandeloo, p. 198)

De quoi se mêle-t-il ? (Buysse)

Le mouvement intérieur apparaît.

Niets meer, al bekroop hem dan ook de lust. (Vandeloo, p. 194)

Rien de plus, même si l'envie le gagnait. (Buysse)

La modalité du mouvement ou de l'action est indiquée; le français se limite au mouvement ou à l'action.

Wipt met een vlugge sprong op een andere balk... (Claes, p. 60)

Saute d'un bond leste sur une autre poutre... (Plard)

1. Dans Anthologie de la prose néerlandaise, sous la direction de P. Brachin, Paris, AubierMontaigne, «Collection bilingue ». Belgique I (1893-1940): E. Claes, "Floere het Fluwijn » (extrait de Floere het Fluwijn), trad. H. Plard, p. 58-72; F. de Pillecijn, "Een soldaat keert terug " (extrait de De Soldaat Johan), trad. P. Brachin, p. 72-88; C. Buysse, "Verval " (extrait de Het Bolleken), trad. L. Fessard. Belgique II (1940-1968) : J. Vandeloo, "Een zonderlinge reis » (extrait de Het Gevaar), trad. M. Buysse, p. 194-217.

2. A. Malblanc, Stylistique comparée du français et de l'allemand, Paris, Didier, 1963, p. 285. Cf. aussi : Blake T. Hanna, "Patterns of Thought in English Translation", META, XIV, $3(1969): 141$. 
Dan stapte de soldaat Johan in de halflichte vroeg-morgen naar buiten. (Pillecijn, p. 76)

Jean le soldat sortit dans la lumière grise du petit matin. (Brachin)

Hij kroop diep weg in het nest... (Claes, p. 60)

Il se retira tout au fond du nid... (Plard)

Le néerlandais décrit des faits; le français les interprète.

En de oude boer knikte. (Pillecijn, p. 76)

Et le vieux paysan approuvait de la tête. (Brachin)

Hij doet of hij een enquête moet houden... (Vandeloo, p. 198)

Se croit-il chargé d'enquêter... (Buysse)

L'écrivain néerlandais met l'accent sur l'individu, sur le cas concret; la représentation française équivalente est plus anonyme, plus impersonnelle.

En [hij] begreep wel hoe onoverkomelijk hij zich mishuwelijkt had. (Buysse, p. 270)

Et [il] comprenait combien sa mésalliance était irréparable. (Fessard)

En hij wankelde bij de eerste voorzichtige stappen... (Pillecijn, p. 72)

Ses premiers pas furent aussi incertains que prudents... (Brachin)

La construction participiale française donne une représentation plus statique, moins dynamique de la réalité.

Waarvan hij de verschijnselen eerst bespeurde nadat ze zich in vastheid van feiten en gewoonten hadden omgezet. (Buysse, p. 264)

Dont il ne constatait les symptômes qu'une fois fixés en faits et en habitudes. (Fessard)

Het was nooit iets geweldigs dat plotseling... oprees... (Buysse)

Ce n'était jamais rien de brusque, surgissant soudain... (Fessard)

Ch. Bally et G. Galichet ${ }^{3}$ soulignent l'importance croissante du style nominal en français. Le verbe néerlandais est souvent traduit par un verbe + substantif.

Hij zag hoe de zwarte raaf behoedzaam om zich heen keek. (Vandeloo, p. 196)

Benting vit le corbeau jeter un regard circulaire et prudent. (Buysse)

Toen hij opzag... (Pillecijn, p. 72)

Lorsqu'il leva les yeux... (Brachin)

En de soldaat Johan sprak. (Pillecijn, p. 76)

Et Jean le soldat prit la parole. (Brachin)

Ailleurs, le syntagme verbal a pour équivalent un syntagme nominal.

Ze waren te mooi, meende zij. (Buysse, p. 266)

Elles étaient trop belles, à son avis. (Fessard)

Juist maar nemen waar hij recht op had. (Vandeloo, p. 194)

N'exiger que son dâ. (Buysse)

Hij kneep zijn ogen halfdicht en keek door de schietgaten naar Wagner. (Vandeloo, p. 200)

Les yeux mi-clos, il regarde Wagner par les meurtrières. (Buysse)

De ce qui précède il faut se garder de conclure que tout syntagme verbal est plus proche de la réalité concrète que tout syntagme nominal. Tout syntagme nominal n'est pas abstrait pour autant. On constate cependant que, lorsque dans la traduction un syntagme verbal néerlandais a pour équivalent un syntagme nominal, la représentation de la réalité est moins dynamique.

b) Le substantif néerlandais serre la réalité tangible de plus près : il est souvent plus précis, plus haut en couleur que l'équivalent français.

3. Charles Bally, Traité de stylistique française, 2 vol., $4^{e}$ éd., Genève, Georg et Cie, 1963; G. Galichet, Grammaire structurale du français moderne, $2^{\mathrm{e}}$ éd., Paris, Hatier, 1968. 
De zware rit van de paarden... (Pillecijn, p. 72)

Le bruit pesant d'une chevauchée... (Brachin)

In de verte hoorde hij doodskreten. (Pillecijn, p. 72)

Au loin il entendait des cris de détresse. (Brachin)

Le traducteur a résumé le syntagme néerlandais; il glisse sur certains détails matériels.

Door de glazen van zijn zonnebril... (Vandeloo, p. 194)

$A$ travers ses lunettes noires... (Buysse)

Een kruising van dominee en misdadiger... (Vandeloo, p. 196)

Mi-pasteur protestant, mi-criminel... (Buysse)

L'infinitif substantivé saisit davantage l'action sur le vif que le substantif.

Het slurpen en het smekken... bleek onuitroeibaar. (Buysse, p. 269)

Il s'avéra impossible de venir à bout de ses lappements et claquements de langue. (Fessard)

L'écrivain néerlandais peint des objets inanimés; le traducteur français souligne le processus de l'action en attirant l'attention sur l'agent.

Bloed was aan zijn mouw in klonters vastgevroren... (Pillecijn, p. 72)

Sur sa manche, le froid avait fixé des caillots de sang... (Brachin)

Comme nous l'avons déjà constaté pour des syntagmes verbaux, le néerlandais se contente de décrire là où le français interprète.

Er stonden reeds 15 of 20 mensen op de volgende trein te wachten. (Vandeloo, p. 200)

Quinze ou 20 personnes attendent déjà le départ suivant. (Buysse)

c) L'adjectif néerlandais contient un élément pictural que n'a pas l'adjectif français.

En hoorde zijn tred in de vriesklare stilte... (Pillecijn, p. 72)

Entendit ses pas résonner dans le silence cristallin [de 1'hiver]... (Brachin)

Si nous prenons comme critères ces différentes caractéristiques, nous pouvons nous demander si l'ensemble d'un texte néerlandais a un caractère plus concret que sa traduction française. Des sondages effectués sur des échantillons de 1000 mots donnent les résultats suivants :

\begin{tabular}{lccc}
\hline & $\mathrm{N}>\mathrm{F}$ & $\mathrm{N}=\mathrm{F}$ & $\mathrm{N}<\mathrm{F}^{*}$ \\
\hline $\begin{array}{l}\text { F. de Pillecijn/P. Brachin } \\
\text { (De Soldaat Johan) }\end{array}$ & 26 & 5 & 5 \\
$\begin{array}{l}\text { J. Vandeloo/M. Buysse } \\
\text { (Het Gevaar) }\end{array}$ & 16 & - & 9 \\
$\begin{array}{l}\text { E. Claes/H. Plard } \\
\text { (Floere het Fluwijn) }\end{array}$ & 9 & 17 & 13 \\
\hline
\end{tabular}

* Les signes $>$, $=,<$, signifient ici : plus concret que, aussi concret que, moins concret que.

Dans la traduction de P. Brachin il y a 10 syntagmes concrets : cinq \& collent 》 au néerlandais; cinq autres viennent du traducteur. Par contre, 26 expressions concrètes ne furent pas rendues en français. M. Buysse a trouvé elle-même neuf représentations concrètes, mais pour 16 autres expressions concrètes néerlandaises il n'y a pas d'équivalents en français.

La traduction de $\mathrm{H}$. Plard foisonne de notations concrètes (30) : des 26 syntagmes concrets du texte néerlandais, 17 furent rendus par des équivalents 
également concrets; de plus la traduction française contient 13 autres syntagmes concrets.

Si nous plaçons le nombre de syntagmes concrets contenus dans les textes néerlandais en face du nombre de syntagmes concrets dans les traductions françaises, nous pouvons dresser le tableau suivant :

\begin{tabular}{lcc}
\hline & néerlandais & français \\
\hline $\begin{array}{l}\text { F. de Pillecijn/P. Brachin } \\
\text { (De Soldaat Johan) }\end{array}$ & 31 & 10 \\
$\begin{array}{l}\text { J. Vandeloo/M. Buysse } \\
\text { (Het Gevaar) }\end{array}$ & 16 & 9 \\
$\begin{array}{l}\text { E. Claes/H. Plard } \\
\text { (Floere het Fluwijn) }\end{array}$ & 26 & 30 \\
\hline
\end{tabular}

Les traductions de $\mathrm{P}$. Brachin et $\mathrm{M}$. Buysse sont moins «concrètes» que l'original; celle de H. Plard l'est davantage. Je ne crois pas que cela soit un effet du hasard. Le traducteur désire non seulement se faire l'interprète des idées de l'auteur néerlandais, mais encore rendre les lecteurs francophones sensibles à la couleur, au réalisme du texte néerlandais.

On peut se demander si un traducteur a une manière de traduire ou si le genre d'œuvre qu'il traduit peut rendre sa langue plus ou moins abstraite. Est-ce que la langue de P. Brachin par exemple est plus concrète (ou abstraite) quand il traduit la prose de A. Vermeylen?

J'ai pris une autre série d'échantillons dans des textes qui ont également été traduits par P. Brachin, M. Buysse et $\mathbf{H}$. Plard. Ce sont ${ }^{4}$ : A. Vermeylen/P. Brachin, Vlaamse Beweging en Europese Geest; Y. Michiels/M. Buysse, Het Boek Alfa; W. Elsschot/H. Piard, Het Algemeen Wereldtijdschrift.

Le tableau de l'analyse contrastive s'établit comme suit :

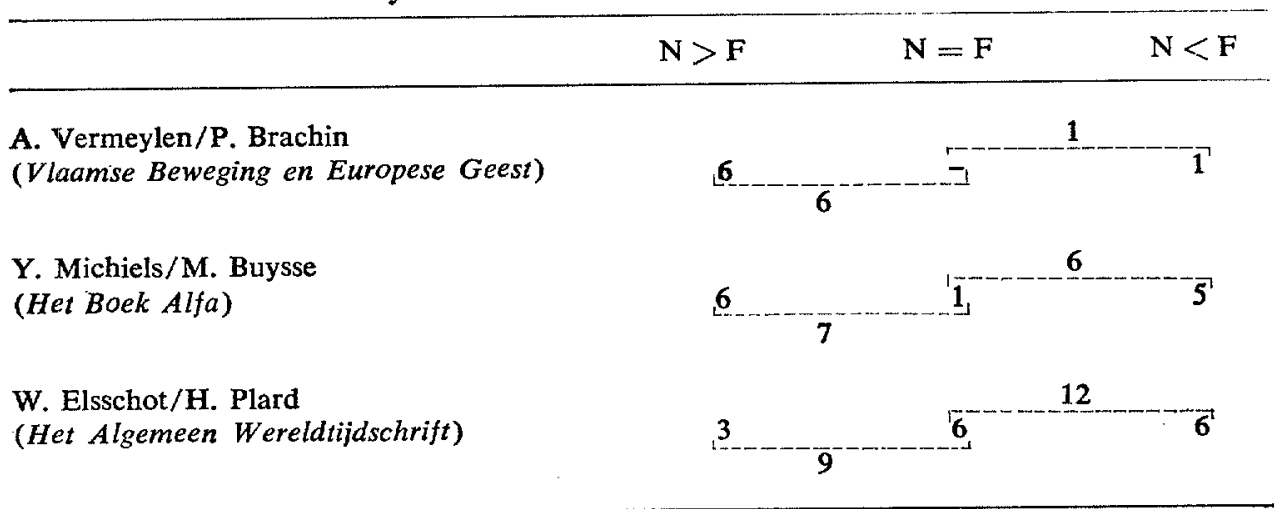

4. Dans Anthologie de la prose nécrlandaise, Belgique I (1893-1940): A. Vermeylen, * Vlaamse Beweging en Europese Geest» (extrait de Vlaamse en Europese Beweging), trad. P. Brachin; W. Elsschot, "Het Algemeen Wereldtijdschrift " (extrait de Lijmen), trad. H. Plard. Y. Michiels, Het Boek Alfa, Amsterdam, De Bezige Bij, et Anvers, Ontwikeling, 1963; trad. M. Buysse, le Livre Alpha, Paris, Gallimard, NRF, 1967. 
Cette deuxième série de textes est écrite dans un néerlandais moins concret que les autres textes. Ce caractère abstrait est encore accusé dans la traduction de P. Brachin. Si celle de M. Buysse est aussi concrète que l'original, la distribution des expressions concrètes y est différente. $\mathrm{H}$. Plard réussit à trouver des équivalents français qui accentuent la plasticité des images néerlandaises.

Le passage d'une langue à une autre n'est donc pas uniquement l'ajustement plus ou moins harmonieux de leurs moyens d'expression; le traducteur peut aussi modeler la langue d'arrivée sur la langue de départ, faisant passer à la fois le contenu et le style du message.

Alors on peut se demander si deux traductions d'un même texte peuvent être essentiellement différentes et si, dans ce cas, cette différence peut s'expliquer, entre autres, par la personnalité du traducteur. Afin d'évaluer l'importance de ce facteur, j'ai comparé des extraits $( \pm 1000$ mots) de deux traductions du Soldaat Johan: celle de P. Brachin et celle de P. de Man ${ }^{5}$.

Voici le résultat du sondage :

\begin{tabular}{|c|c|c|c|}
\hline & $N>F$ & $\mathbf{N}=\mathbf{F}$ & $\mathbf{N}<\mathbf{F}$ \\
\hline P. Brachin & 13 & 2 & 2 \\
\hline P. de Man & {$[5+2]$} & $8 / 0$ & 0 \\
\hline
\end{tabular}

Les deux traductions sont plus abstraites que le texte original. La traduction de P. de Man contient huit syntagmes concrets, celle de P. Brachin, quatre. Treize expressions néerlandaises sont plus concrètes que les équivalents de P. Brachin; P. de Man en a traduit huit de façon aussi concrète qu'en néerlandais.

Bloed was aan zijn mouw in klonters vastgevroren... (Pillecijn, p. 72)

Sur sa manche, le froid avait fixé des caillots de sang... (Brachin, p. 73)

Du sang s'était coagulé en caillots sur sa manche... (P. de Man, p. 8)

In het woud slachtten de boeren de soldeniers af. (Pillecijn, p. 72)

Dans la forêt, les paysans assommaient les mercenaires. (Brachin, p. 73)

Parmi les arbres, les paysans abattaient la soldatesque! (P. de Man, p. 8)

Het groeiende leven van het vee... (Pillecijn, p. 76)

La croissance de la vie chez les animaux... (Brachin, p. 77)

La vie grandissante du bétail... (P. de Man, p. 10)

Mais là où la traduction de $\mathrm{P}$. Brachin est au moins aussi concrète que le texte néerlandais $(\mathrm{N}=\mathrm{F}: 2 \mathrm{x} ; \mathrm{N}<\mathrm{F}: 2 \mathrm{x}$ ), la traduction de $\mathrm{P}$. de Man est plus générale.

P. de Man serre le texte original de plus près, par exemple lorsqu'il traduit :

De koe die gelaten en stil haar jong zoogde. (Pillecijn, p. 76)

La vache qui, résignée et silencieuse, allaitait son petit. (P. de Man, p. 11)

$\mathrm{La}$ vache qui, tranquille et silencieuse, allaitait son petit. (Brachin, p. 77)

Hij lag met zijn gezicht tegen de grond en alles werd dof boven hem... (Pillecijn, p. 72)

Il était couché, la face au sol et tout s'assourdit au-dessus de lui... (P. de Man, p. 7)

Il se retrouva la figure contre le sol, et tout se voila au-dessus de lui... (Brachin, p. 73)

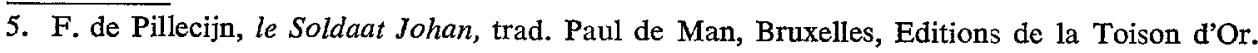


mais c'est parfois aux dépens de la correction :

Dit was de nacht van Driekoningen... (Pillecijn, p. 74)

C'était la nuit des Trois-Rois... (P. de Man, p. 8)

C'était la nuit des Rois... (Brachin, p. 75)

L'emploi plus ou moins concret de la langue d'arrivée peut donc être expliqué par la personnalité du traducteur et ses intentions stylistiques. Le traducteur doit s'efforcer de combler l'écart qui existe entre deux personnalités : la sienne et celle de l'auteur. Lorsque Thomas Mann invitait sa traductrice anglaise, Mrs. Helen Tracy Lowe-Porter ${ }^{6}$, à lire les œuvres qui avaient influencé tel roman qu'il lui proposait, il attendait d'elle qu'elle s'intègre dans son monde à lui, qu'elle devienne son alter ego, non pas afin de transposer son ouvrage avec la plus grande précision possible, mais bien afin de le réécrire en anglais.

La dualité auteur/traducteur n'existe plus si l'auteur néerlandais transpose lui-même ses écrits en français. On peut se demander si un auteur néerlandais au style imagé choisit des équivalents également concrets en français ou si le seul fait que la langue d'arrivée soit le français est suffisant pour motiver une traduction plus abstraite.

On peut admettre que le Livre de Joachim ${ }^{7}$ de Jan Albert Goris soit une version française de Joachim van Babylon de Marnix Gijsen, pseudonyme de J.A. Goris. Certes on ne mentionne nulle part qu'il s'agit d'une transposition d'un roman néerlandais. Toutefois à l'analyse il m'est apparu que la version française est bien la traduction de l'original néerlandais. exemple :

J. A. Goris a traduit Marnix Gijsen de la manière suivante $: N>F: 15 x$, par

Ook hij volgde Daniel's tekst op den voet... (Gijsen, p. 6)

Lui aussi se servit du texte de Daniel... (Goris, p. 10)

En brandmerkte de houding der rechters... (Gijsen, p. 7)

Il dénonça [...], les mauvais juges... (Goris, p. 10)

$N=F: 3 x$.

$N<F: 3 x$, par exemple :

De schildering van de ondeugd is natuurlijk altijd dankbaarder dan de afbeelding der deugd... (Gijsen, p. 6)

Évidemment, dépeindre le vice est toujours plus facile que dépeindre la vertu... (Goris, p. 10)

Ce qui frappe, c'est l'adaptation de la traduction destinée à un public différent par l'emploi d'images dont les connotations sont susceptibles d'en appeler davantage à des lecteurs français.

Een echte Spartaan... een zin uit de Genesis... en zoo meer... (Gijsen, p. 7)

Un vrai Spartiate... une phrase digne de la Bible... (Goris, p. 11)

Stel u voor dat in Jerusalem een naaikransje bestaan zou van de weduwen van Koning David..

(Gijsen, p. 8)

Imaginez un cénacle des veuves du roi David... (Goris, p. 12)

6. J.C. Thirlwall, In Another Language, A Record of the Thirty-year Relationship between Thomas Mann and His American Translator, Helen Tracy Lowe-Porter, New York, Alfred A. Knopf, 1966 , p. 22.

7. Marnix Gijsen, Joachim van Babylon, La Haye, A.A.M. Stols; trad. J.A. Goris, le Livre de Joachim de Babylone, Anvers, La Haye, Luxembourg, A.A.M. Stols, et Paris, Nouvelles Editions latines, 1950. 
Haar muiltjes en haar eersten kruisjessteek uit school... (Gijsen, p. 8)

Ses mules roses et sa première feuille d'écriture... (Goris, p. 12)

Le Livre de Joachim contient plus de détails. Cette «explicitation », selon la terminologie de A. Malblanc (p. 6), est peut-être due à la crainte inconsciente du traducteur de ne pas toujours se faire comprendre.

En dan nog op een tekst waarvan ze blijkbaar niets begrepen... (Gijsen, p. 7)

Et puis elles chantaient un texte auquel manifestement elles ne comprenaient goutte, mais je me trompe peut-être sur les filles d'aujourd'hui... (Goris, p. 11)

Opdat het drama zijn volle betekenis krijge, moeten al de spelers onbereikbaar zijn. (Gijsen, p. 8)

Pour que le drame atteigne son plein sens et une forme qui lui assure l'éternité, il faut que tous ses protagonistes, comme ses témoins, aient disparu. (Goris, p. 11)

Il semble donc que dans tous les sondages effectués $(\mathrm{N} \longrightarrow \mathrm{F})$ un texte original néerlandais «concret» devienne plus ou moins «abstrait» en français; le degré d'abstraction dépend toutefois de la personnalité du traducteur et du choix (conscient ou non) qu'il fait des moyens d'expression de la langue d'arrivée.

Pouvons-nous renverser cette constatation et dire qu'inversement un texte écrit en français devient plus concret lorsqu'on le traduit en néerlandais?

J'ai comparé des extraits (environ 1000 mots) des ouvrages suivants et de leur traduction ${ }^{8}$ : A. Camus, l'Étranger, trad. A. Morriën ; A. Camus, le Mythe de Sisyphe, trad. C.N. Lijsen ; A. Malraux, la Condition humaine, trad. E. du Perron; F. Sagan, Un certain sourire, trad. H. Lampo.

L'analyse contrastive fournit les données numériques suivantes :

\begin{tabular}{lccc}
\hline & $\mathrm{N}>\mathrm{F}$ & $\mathrm{N}=\mathrm{F}$ & $\mathrm{N}<\mathrm{F}$ \\
\hline $\begin{array}{l}\text { A. Malraux/E. du Perron } \\
\text { (la Condition humaine) }\end{array}$ & 20 & - & 2 \\
$\begin{array}{l}\text { A. Camus/A. Morriën } \\
\text { (l'Étranger) }\end{array}$ & 11 & 1 & 4 \\
$\begin{array}{l}\text { F. Sagan/H. Lampo } \\
\text { (Un certain sourire) }\end{array}$ & 11 & - & 4 \\
$\begin{array}{l}\text { A. Camus/C. N. Lijsen } \\
\text { (le Mythe de Sisyphe) }\end{array}$ & 2 & - & \\
\hline
\end{tabular}

Les traductions néerlandaises sont plus concrètes que les textes originaux.

Tchen avait l'impression de le tenir fixé au lit... (Malraux, p. 9)

Kreeg Tsjen de indruk dat hij het op bed genageld hield... (E. du Perron, p. 9)

D'un coup à traverser une planche... (Malraux, p. 9)

Met een stoot om een plank te doorboren... (E. du Perron, p. 9)

J'avais rarement le goût de prendre... (Sagan, p. 23)

Zelden bekruipt mij de lust om mij meester van iets te maken... (Lampo, p. 16)

8. A. Camus, l'Etranger, Paris, Gallimard, 1957; trad. A. Morriën, De Vreemdeling, Amsterdam, De Bezige Bij, 1967. A. Camus, le Mythe de Sisyphe, Paris, Gallimard, NRF, 1942; trad. C.N. Lijsen, De Myte van Sisyfus, Amsterdam, De Bezige Bij, 1967. A. Malraux, la Condition humaine, Paris, Gallimard, 1946; trad. E. du Perron, Het Menselijk Tekort. Amsterdam, Anvers, Wereld-Bibliotheek, 1956. F. Sagan, Un certain sourire, Paris, Julliard, 1959; trad. H. Lampo, Een verre Glimlach, Bruxelles, La Haye, A. Manteau, 1965. 
Comme le magazine avait une douzaine de pages... (Camus, p. 67)

Aangezien het blad een twaalftal pagina's dik was... (Morriën, p. 54)

La vieillesse ne se guérit pas... (Camus, p. 70)

Tegen de ouderdom is geen kruid gewassen... (Morriën, p. 56)

$\mathrm{La}$ réalité matérielle est plus présente chez E. du Perron que chez A. Morriën par exemple. Le mode de représentation dans la langue d'arrivée, ici le néerlandais, n'est pas seulement conditionné par la langue elle-même, mais aussi par l'usage que le traducteur en fait.

De cette analyse on peut donc conclure que le néerlandais a une préférence pour les expressions concrètes, le français pour les expressions plus abstraites. Il serait erroné d'en déduire que le français ne possède pas d'équivalents colorés, proches de la vie. On ne peut que constater que la représentation française est généralement plus abstraite.

Si une traduction française évoque la réalité néerlandaise avec le même sens des choses matérielles, comme c'est le cas dans les traductions de H. Plard, c'est que le traducteur utilise volontairement la langue d'arrivée (ici le français) d'une manière telle qu'elle transmette non seulement les idées, mais encore certaines qualités de la langue de départ (ici le néerlandais).

Marie-Jeanne De VRiendt-De Man 